\title{
Long-Term Course of Brain-Derived Neurotrophic Factor Serum Levels in a Patient Treated with Deep Brain Stimulation of the Lateral Habenula
}

\author{
Carolin Hoyer $^{\mathrm{a}}$ Laura Kranaster $^{\mathrm{a}} \quad$ Alexander Sartorius $^{\mathrm{a}} \quad$ Rainer Hellweg $^{\mathrm{b}}$ \\ Peter Gass ${ }^{\mathrm{a}}$ \\ ${ }^{a}$ Department of Psychiatry and Psychotherapy, Central Institute of Mental Health (CIMH), Medical Faculty Mannheim, \\ University of Heidelberg, Mannheim, and ${ }^{\text {b}}$ Department of Psychiatry, Charité Campus Mitte, Berlin, Germany
}

\section{Key Words}

Brain-derived neurotrophic factor · Depression $\cdot$ Deep

brain stimulation $\cdot$ Habenula

\begin{abstract}
Introduction: According to the neurotrophin hypothesis, a brain-derived neurotrophic factor (BDNF) decrease has been postulated as a pivotal pathomechanism in affective disorder, and the treatment-associated increase in peripheral BDNF has been linked to therapeutic efficacy of antidepressant drugs and electroconvulsive therapy. However, in deep brain stimulation (DBS), a still experimental antidepressant treatment approach, this issue has not yet been investigated. Methods: We examine the long-term course of serum BDNF levels in a 64-year-old woman who is being treated with DBS of the lateral habenula for severe major depressive disorder. Results: Our main findings are a significant increase in BDNF serum levels following DBS of the lateral habenula and an inverse U-shaped correlation of depression scores and BDNF levels. Discussion: The data indicate that DBS, like other effective antidepressant treatments, may contribute to an increase in peripheral BDNF levels, which are thought to reflect central nervous DBS-induced neuroplastic changes. Moreover, our observations underscore the complex nature
\end{abstract}

of disease-associated BDNF alterations. Their identification as either state or trait marker remains controversial and requires larger-scale longitudinal studies.

Copyright $\odot 2012$ S. Karger AG, Basel

\section{Introduction}

Brain-derived neurotrophic factor (BDNF) is involved in the promotion of neuronal growth, the maintenance of synaptic functioning and neural plasticity as well as neuronal repair and survival $[1,2]$. The 'neurotrophin hypothesis' of depression postulates a stress-induced or stress-associated BDNF decrease as a pivotal pathomechanism of affective disorders such as major depressive disorder (MDD) or bipolar disorder [3-5]. Both antidepressant medication and electroconvulsive therapy (ECT) induce an increase in both central and peripheral BDNF levels, suggesting that the amelioration of depressive symptoms in the wake of these therapeutic regimens is at least partly modulated by BDNF [6-8]. Direct delivery of

C. Hoyer, L. Kranaster and A. Sartorius contributed equally as first authors. R. Hellweg and P. Gass contributed equally as senior authors.

\section{KARGER}

Fax +41613061234

E-Mail karger@karger.ch

www.karger.com (c) 2012 S. Karger AG, Basel

0302-282X/12/0653-0147\$38.00/0

Accessible online at:

www.karger.com/nps
Alexander Sartorius, $\mathrm{MD}, \mathrm{PhD}$

Central Institute of Mental Health

Ruprecht Karl University Heidelberg, J5

DE-68159 Mannheim (Germany)

Tel.+49 621170 30, E-Mail alexander.sartorius@zi-mannheim.de 


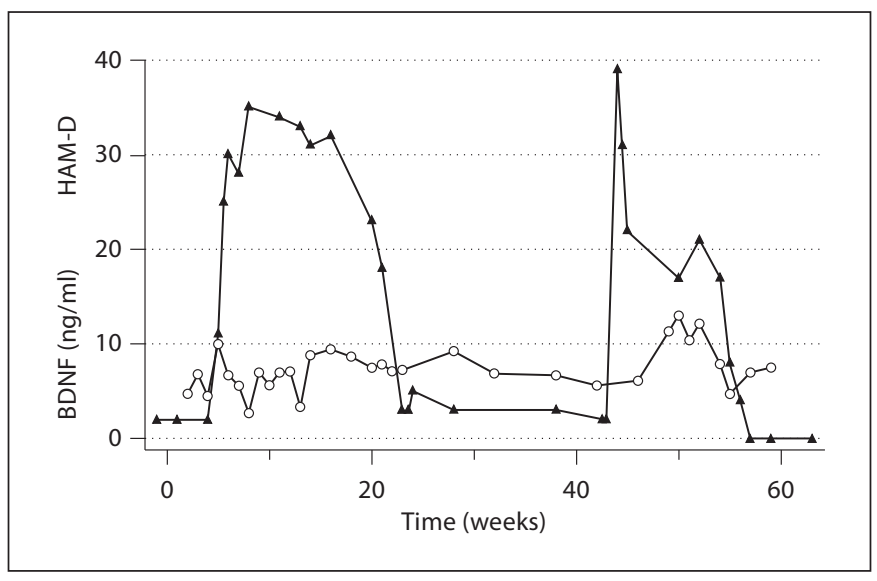

Fig. 1. Longitudinal course of the patient's HAM-D scores $(\boldsymbol{\Delta})$ and corresponding serum BDNF levels $(\bigcirc)$.

BDNF into the hippocampus of rats [9-11] and mice [12] has shown an antidepressant effect in animal models of depression; these effects in turn were blocked when a forebrain-specific knockout of the BDNF-encoding gene was performed [13]. Thus, it seems tempting to employ the serum BDNF level as an easily accessible biomarker indicating a state of affective imbalance or, more generally, stress [14, 15]. However, the straightforward measurement and interpretation of serum BDNF is impeded by several factors: (1) platelets are known to store BDNF and are able to release it in response to certain triggers [16]; (2) the serum BDNF concentration is up to 50 -fold higher than the plasma concentration, thus serum levels basically reflect the platelet depot [17]; (3) it is uncertain whether the peripherally measured levels truly represent the central status. However, it has been demonstrated in preclinical studies that central and peripheral levels correlate in a sufficient way, but with a time delay of the magnitude of a platelet's lifespan [18-20].

The recent adoption of deep brain stimulation (DBS) for psychiatric disease such as obsessive-compulsive disorder, Tourette's syndrome or major depression has already shown efficacy in a well-chosen, highly treatmentresistant collective [21-23]. While clinical results for patients suffering from chronic MDD are promising, the currently still experimental nature of DBS calls for a comprehensive analysis of mechanisms alongside the evaluation of antidepressant efficacy. So far, the question whether DBS may influence BDNF levels has not yet been investigated. Since increased BDNF expression has been found in the wake of ECT or treatment with antidepressant medication, both in preclinical and clinical studies,

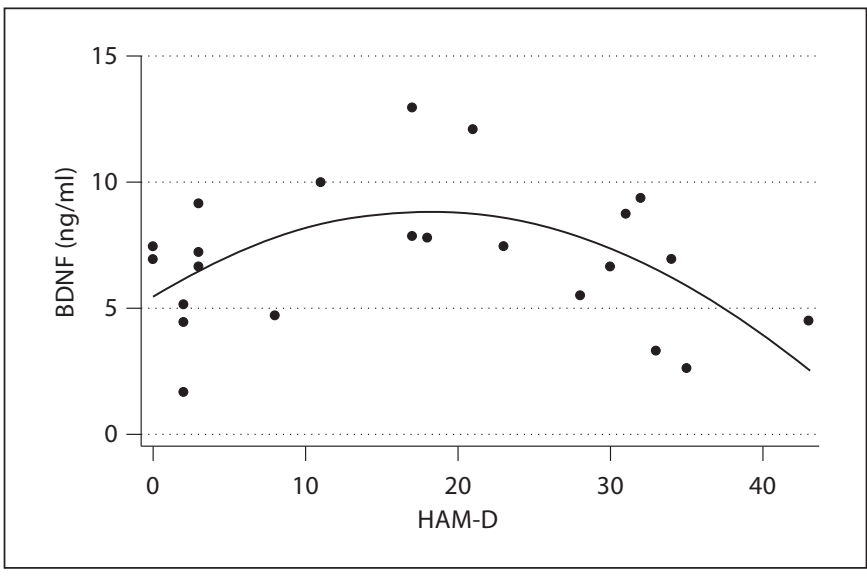

Fig. 2. Regression analysis depicting the correlation between HAM-D scores and serum BDNF.

we assume that DBS - if effective in treatment-resistant major depression - will also lead to an increase in BDNF. Here we provide case data from a female patient with major depression who received DBS of the lateral habenula, a new target for DBS [24].

\section{Methods}

The case has been described in greater detail elsewhere [25]. In brief, a 64-year-old female patient had been suffering from major depressive episodes since the age of 18 without any signs of bipolarity. Appropriate pharmacotherapy including antidepressants of different modes of action, as well as combination and augmentation strategies, could not control the illness. At the age of 59 she received ECT for the first time. Although remission was achieved, it was not stable. During maintenance ECT, which was initiated after several relapses, it was impossible to extend between-session intervals to more than 2 weeks without relapse despite concomitant medication. With deteriorating quality of life and increasing ECT- and pharmacotherapy-associated side effects, DBS surgery was performed in July 2008 in order to dampen an assumed overactivation of the lateral habenula, which, through its reciprocal connections with dopaminergic and serotonergic neurons, has been implicated in the pathophysiology of depression [26, 27]. DBS treatment resulted in a sustained full remission of depressive symptoms in our severely ill patient. Interestingly, she suffered from a relapse when stimulation was accidentally discontinued (fig. 1). In the 60 weeks following surgery, the patient was closely monitored every other week. Due to the severity of the illness, she received psychotropic medication during the observation period. She received risperidone, sertraline and mirtazapine at stable doses. During week 16, clinical management necessitated a switch from sertraline to venlafaxine and the re-introduction of lithium during week 17. From that time onwards, medication remained unchanged. During the biweekly visits, we performed clinical examinations and ratings with the Hamilton Rating Scale for De- 
pression (HAM-D) as well as BDNF serum level measurement. The time course of BDNF serum levels and symptom severity measured by the HAM-D is displayed in figure 1. In figure 2 we show BDNF levels in relation with HAM-D scores.

\section{Method of Serum BDNF Measurement and}

Statistical Analysis

Samples of venous blood were taken in additive-free serum containers and were left to clot for about $1 \mathrm{~h}$, followed by centrifugation at 4,000 rpm for $4 \mathrm{~min}$ at room temperature. Serum samples were stored at $-80^{\circ} \mathrm{C}$ until analysis. BDNF levels in serum were quantified as described in detail earlier [28]. The neurotrophin levels were expressed as nanograms per millilitre. Special care was taken to minimize the influence of unavoidable variances between experiments [29]. Regression analysis and t tests were performed using Stata ${ }^{\circledR}$ (Stata Corp, Tex., USA, version 11) at a significance level of 0.05 .

\section{Results}

\section{Initially Lower BDNF Levels}

As it can be seen in figure 1, an obvious step of BDNF levels can be detected after week 13 (DBS started at week 1). The mean of all measured BDNF levels until week 13 is $5.8 \pm 2.0 \mathrm{ng} / \mathrm{ml}$, the mean of all BDNF levels later on $8.2 \pm 2.2 \mathrm{ng} / \mathrm{ml}$. This increase is significant, even if only weeks $0-13$ and weeks $14-45$ are compared $(\mathrm{p}=0.0001$ vs. $\mathrm{p}=0.001$, respectively, for a two-sided t test). BDNF levels taken from 9 weeks to 3 weeks before DBS surgery had been even lower, i.e. $3.7 \pm 1.7 \mathrm{ng} / \mathrm{ml}$ ( $\mathrm{n}=6$ measurements).

\section{Inverse U-Shaped Correlation of HAM-D and BDNF Serum Levels}

As can be seen in figure 2, an inverse U-shaped correlation of HAM-D and BDNF can be derived from our data. To model an inverse U-shaped relation, we used the simplest mathematical assumption, which is a secondorder polynomial regression. A regression analysis (as shown in fig. 2) yields a significant correlation at $\mathrm{p}=0.035$ $\left(\mathrm{n}=23, \mathrm{r}^{2}=0.21\right)$. Since we also detected a linear correlation between BDNF serum levels and thrombocytes per nanolitre, we included platelet count as a covariate into the regression analysis. The covariate influence was not significant, however $(\mathrm{p}=0.12)$.

\section{Discussion}

Here we describe for the first time long-term changes in BDNF serum levels in a patient who had undergone DBS of the lateral habenula for treatment-resistant MDD.
Our data corroborate findings of serum BDNF increase under efficient antidepressive treatments such as antidepressant pharmacotherapy and ECT $[14,30]$. While we are unaware of studies investigating the course of BDNF in the wake of DBS treatment for depression in a systematic way, it was previously shown that the antidepressant effect observed after programmed acute electrical stimulation of the ventral tegmental area in a rat genetic model of depression was accompanied by increases in BDNF mRNA and protein levels in the prefrontal cortex and the nucleus accumbens [31]. Furthermore, the temporally shifted correlation with symptom severity in this study is in line with the idea that a time lag of necessity exists for central BDNF to come to an equilibrium with peripheral BDNF levels in the platelet pool, as suggested previously by a systematic time course of central and peripheral BDNF levels after chronic ECT in rats [20]. Similarly, significant increases in serum BDNF levels were found in ECT-treated patients 1 month after cessation of the procedure while no changes had been noted immediately after the last session [32]. Thus, the central BDNF level change presumably happens first, followed by a relatively fast improvement of psychopathology and a delayed increase in peripheral BDNF levels [20]. Finally, neurons of the lateral habenula project to the ventral tegmental area and the dorsal raphe nuclei, which are critically involved in the release of dopamine and serotonin, respectively $[26,27]$. Since serotonin impacts on BDNF expression and genetic epistasis of the two systems has been observed in different species [33], habenular functional modulation through DBS may thus indirectly influence BDNF.

While the BDNF elevation certainly cannot be exclusively attributed to DBS action in our case, the observed time lag nonetheless supports the notion that DBS contributed to BDNF level alterations. In addition, the long and severe disease history renders placebo response [34] or natural remission rather unlikely.

The low peripheral BDNF our patient presented with immediately prior to DBS, when severe symptoms were present, would argue for BDNF decrease as a state marker for depression. The observed rise of serum BDNF under efficient antidepressant treatment would also be in accordance with this. However, levels did not remain stably elevated but even though the patient reached a sustained formal remission, serum BDNF returned to lower levels. Given the long psychiatric history of our patient, there is certainly a high intrinsic vulnerability for MDD. Along these lines, low peripheral BDNF is apparently not limited to phases of acute disease but has also been ob- 
served in euthymic MDD or bipolar disorder patients [35] as well as bipolar mania [36], in healthy subjects with depression-related personality traits [37] and bipolar disorder patients with adverse childhood experiences [38]. The identification of the nature of low BDNF levels as either a state marker or an endophenotypic trait is thus not straightforward. According to Kapczinski et al. [39], the pattern of BDNF levels in patients with mood disorders should be interpreted 'as a composite of changes related to the trait of having a mood disorder and state changes related to the strain imposed by environmental factors and repeated mood episodes'. We share this view in the sense that the depressive trait obviously goes along with lower BDNF levels and any kind of sufficient antidepressive action raises these levels, resulting in a temporarily delimited BDNF increase, which might thus be required for an antidepressive effect. After this transient increase, BDNF levels then return back to or almost to trait level. The inverse U-shaped relation between symptom severity and BDNF levels from our patient is in line with this view. Alternatively, disease episode-bound BDNF decreases here one would assume BDNF to be a true state marker - may occur repeatedly over a long period of time and lead to dysregulation and lasting imbalance of the system so that a recovery to normal values does not occur (for a recent study invoking an explanation along these lines for decreased BDNF levels in patients in early remission, see Molendijk et al. [40]). In general, there is a paucity of studies investigating long-term BDNF changes in patients suffering from affective disorders.

Interpretations of peripheral BDNF, its exact role, relevance and implications for disease pathophysiology crucially rest on the assumption of serum BDNF truly reflecting cerebral BDNF metabolism. There is substantial evidence in favour of this assumption [18-20,41, 42]; nevertheless, there are a number of important aspects to consider, the first of which concerns blood-brain barrier permeability of BDNF. Findings are equivocal with regard to this question [43]. Second, both peripherally and centrally, mononuclear cells and T cells contribute to BDNF production constitutively as well as upon certain activation signals $[44,45]$. Inflammatory processes have been implied in depression pathophysiology [46], and as they are intimately linked to mononuclear, $\mathrm{T}$ and $\mathrm{B}$ cell activity, changes of BDNF levels in depression may result from altered immune cell activitiy. Third, neurons, the primary source of BDNF in the central nervous system [47], possess distinct mechanisms allowing for a rapid and dynamic regulation of BDNF expression, localization and synaptic delivery [48]. Thus, further characterization of these mechanisms, the ways in which they are potentially impaired in affective disorder and how that may affect peripheral BDNF levels may shed further light upon the relation between central and peripheral states. Decreased peripheral BDNF levels might also result from dysregulated BDNF expression or release by peripheral cells [49], particularly platelets, the function of which has been shown to be altered in depression [50,51]. While we observed a correlation between platelet counts and BDNF levels in our patient, platelet count did not significantly influence our results. A similar correlation, not confounded by the co-occurrence of a psychiatric disorder such as depression or dementia, was previously found in a sample of elderly individuals [28]. Last but not least, glucocorticoids inhibit BDNF production [52], and hypercortisolism in depression may accordingly contribute to a disease-associated decrease in BDNF. We are well aware of the inherent limitations of a case study like the one presented here, most prominently its retrospective and descriptive character, whereby it lacks experimental control, and issues of generalizability. However, case observations are a valuable means of generating hypotheses and collecting information. This is especially relevant with regard to as yet experimental therapeutic methods such as DBS for the treatment of affective disorders, for which there is still limited clinical experience [23] and currently only one follow-up study [22].

In summary, this is the first case of a patient suffering from MDD with longitudinal alterations of BDNF under concurrent DBS. Beyond the impressive clinical outcome of the patient, the course of BDNF levels also supports our hypothesis that stimulating the habenula could lead to amelioration of symptoms in patients suffering from treatment-resistant severe MDD. In this sense, antidepressive treatment - habenular DBS in our case - may induce neuroplastic changes that are indexed to serum BDNF. Changes in BDNF may even serve as a treatment response marker because the absence of an early increase in peripheral BDNF has been shown to predict non-response to antidepressant therapy [53]. The measurement of serum BDNF levels following the course of illness for a considerable time during all phases of the disease is certainly an important prerequisite to further elucidate the role of BDNF in affective disorder and gain insight into mechanisms of effective antidepressant treatment options. 


\section{References}

$>1$ D'Sa C, Duman RS: Antidepressants and neuroplasticity. Bipolar Disord 2002;4:183194.

$>2$ Lewin GR, Barde YA: Physiology of the neurotrophins. Annu Rev Neurosci 1996;19: 289-317.

$\checkmark 3$ Duman RS, Heninger GR, Nestler EJ: A molecular and cellular theory of depression. Arch Gen Psychiatry 1997;54:597-606.

-4 Altar CA: Neurotrophins and depression. Trends Pharmacol Sci 1999;20:59-61.

$>5$ Pittenger C, Duman RS: Stress, depression, and neuroplasticity: a convergence of mechanisms. Neuropsychopharmacology 2008; 33:88-109.

$\checkmark 6$ Matrisciano F, Bonaccorso S, Ricciardi A, Scaccianoce S, Panaccione I, Wang L, Ruberto A, Tatarelli R, Nicoletti F, Girardi P, Shelton RC: Changes in BDNF serum levels in patients with major depression disorder (MDD) after 6 months treatment with sertraline, escitalopram, or venlafaxine. J Psychiatr Res 2009;43:247-254.

7 Nibuya M, Morinobu S, Duman RS: Regulation of BDNF and trkB mRNA in rat brain by chronic electroconvulsive seizure and antidepressant drug treatments. J Neurosci 1995; 15:7539-7547.

$>8$ Piccinni A, Del Debbio A, Medda P, Bianchi C, Roncaglia I, Veltri A, Zanello S, Massimetti E, Origlia N, Domenici L, Marazziti D, Dell'Osso L: Plasma brain-derived neurotrophic factor in treatment-resistant depressed patients receiving electroconvulsive therapy. Eur Neuropsychopharmacol 2009; 19:349-355.

$>9$ Shirayama Y, Chen AC, Nakagawa S, Russell DS, Duman RS: Brain-derived neurotrophic factor produces antidepressant effects in behavioral models of depression. J Neurosci 2002;22:3251-3261.

10 Hoshaw BA, Malberg JE, Lucki I: Central administration of IGF-I and BDNF leads to long-lasting antidepressant-like effects. Brain Res 2005;1037:204-208.

-11 Sirianni RW, Olausson P, Chiu AS, Taylor JR, Saltzman WM: The behavioral and biochemical effects of BDNF containing polymers implanted in the hippocampus of rats. Brain Res 2010;1321:40-50.

$>12$ Gourley SL, Kiraly DD, Howell JL, Olausson P, Taylor JR: Acute hippocampal brain-derived neurotrophic factor restores motivational and forced swim performance after corticosterone. Biol Psychiatry 2008;64: 884-890.

-13 Adachi M, Barrot M, Autry AE, Theobald D, Monteggia LM: Selective loss of brain-derived neurotrophic factor in the dentate gyrus attenuates antidepressant efficacy. Biol Psychiatry 2008;63:642-649.
14 Mossner R, Mikova O, Koutsilieri E, Saoud M, Ehlis AC, Muller N, Fallgatter AJ, Riederer P: Consensus paper of the WFSBP Task Force on Biological Markers: biological markers in depression. World J Biol Psychiatry 2007;8:141-174.

15 Taliaz D, Loya A, Gersner R, Haramati S, Chen A, Zangen A: Resilience to chronic stress is mediated by hippocampal brain-derived neurotrophic factor. J Neurosci 2011; 31:4475-4483.

16 Fujimura H, Altar CA, Chen R, Nakamura T, Nakahashi T, Kambayashi J, Sun B, Tandon $\mathrm{NN}$ : Brain-derived neurotrophic factor is stored in human platelets and released by agonist stimulation. Thromb Haemost 2002; 87:728-734.

17 Radka SF, Holst PA, Fritsche M, Altar CA: Presence of brain-derived neurotrophic factor in brain and human and rat but not mouse serum detected by a sensitive and specific immunoassay. Brain Res 1996;709:122301.

18 Pan W, Banks WA, Fasold MB, Bluth J, Kastin AJ: Transport of brain-derived neurotrophic factor across the blood-brain barrier. Neuropharmacology 1998;37:15531561.

19 Poduslo JF, Curran GL: Permeability at the blood-brain and blood-nerve barriers of the neurotrophic factors: NGF, CNTF, NT-3, BDNF. Brain Res Mol Brain Res 1996;36: 280-286.

20 Sartorius A, Hellweg R, Litzke J, Vogt M, Dormann C, Vollmayr B, Danker-Hopfe H, Gass P: Correlations and discrepancies between serum and brain tissue levels of neurotrophins after electroconvulsive treatment in rats. Pharmacopsychiatry 2009;42:270276.

21 Schlapfer TE, Bewernick BH: Deep brain stimulation for psychiatric disorders - state of the art. Adv Tech Stand Neurosurg 2009; 34:37-57.

22 Kennedy SH, Giacobbe P, Rizvi SJ, Placenza FM, Nishikawa Y, Mayberg HS, Lozano AM: Deep brain stimulation for treatment-resistant depression: follow-up after 3 to 6 years. Am J Psychiatry 2011;168:502-510.

23 Blomstedt P, Sjoberg RL, Hansson M, Bodlund O, Hariz MI: Deep brain stimulation in the treatment of depression. Acta Psychiatr Scand 2011;123:4-11.

24 Sartorius A, Henn FA: Deep brain stimulation of the lateral habenula in treatment resistant major depression. Med Hypotheses 2007;69:1305-1308.

25 Sartorius A, Kiening KL, Kirsch P, von Gall CC, Haberkorn U, Unterberg AW, Henn FA, Meyer-Lindenberg A: Remission of major depression under deep brain stimulation of the lateral habenula in a therapy-refractory patient. Biol Psychiatry 2010;67:e9-e11.
26 Hikosaka O, Sesack SR, Lecourtier L, Shepard PD: Habenula: crossroad between the basal ganglia and the limbic system. J Neurosci 2008;28:11825-11829.

27 Sartorius A, Meyer-Lindenberg A: Deep brain stimulation of the lateral habenula to treat depression. Front Neurosci 2009;3:272.

28 Ziegenhorn AA, Schulte-Herbruggen O, Danker-Hopfe H, Malbranc M, Hartung HD, Anders D, Lang UE, Steinhagen-Thiessen E, Schaub RT, Hellweg R: Serum neurotrophins - a study on the time course and influencing factors in a large old age sample. Neurobiol Aging 2007;28:1436-1445.

29 Hellweg R, Lohmann P, Huber R, Kuhl A, Riepe MW: Spatial navigation in complex and radial mazes in APP23 animals and neurotrophin signaling as a biological marker of early impairment. Learn Mem 2006;13:6371.

30 Brunoni AR, Lopes M, Fregni F: A systematic review and meta-analysis of clinical studies on major depression and BDNF levels: implications for the role of neuroplasticity in depression. Int J Neuropsychopharmacol 2008;11:1169-1180.

31 Friedman A, Frankel M, Flaumenhaft Y, Merenlender A, Pinhasov A, Feder Y, Taler M, Gil-Ad I, Abeles M, Yadid G: Programmed acute electrical stimulation of ventral tegmental area alleviates depressive-like behavior. Neuropsychopharmacology 2009;34: 1057-1066.

32 Bocchio-Chiavetto L, Zanardini R, Bortolomasi M, Abate M, Segala M, Giacopuzzi M, Riva MA, Marchina E, Pasqualetti P, Perez J, Gennarelli M: Electroconvulsive therapy (ECT) increases serum brain derived neurotrophic factor (BDNF) in drug resistant depressed patients. Eur Neuropsychopharmacol 2006; 16:620-624.

33 Martinowich K, Lu B: Interaction between BDNF and serotonin: role in mood disorders. Neuropsychopharmacology 2008;33: 73-83.

34 Mayberg HS, Lozano AM, Voon V, McNeely HE, Seminowicz D, Hamani C, Schwalb JM, Kennedy SH: Deep brain stimulation for treatment-resistant depression. Neuron 2005;45:651-660.

35 Monteleone P, Serritella C, Martiadis V, Maj M: Decreased levels of serum brain-derived neurotrophic factor in both depressed and euthymic patients with unipolar depression and in euthymic patients with bipolar I and II disorders. Bipolar Disord 2008;10:95-100.

36 Fernandes BS, Gama CS, Maria CK, Yatham LN, Fries GR, Colpo G, de Lucena D, Kunz M, Gomes FA, Kapczinski F: Brain-derived neurotrophic factor as a state-marker of mood episodes in bipolar disorders: a systematic review and meta-regression analysis. J Psychiatr Res 2011;45:995-1004. 
-37 Lang UE, Hellweg R, Gallinat J: BDNF serum concentrations in healthy volunteers are associated with depression-related personality traits. Neuropsychopharmacology 2004; 29:795-798.

38 Kauer-Sant'Anna M, Tramontina J, Andreazza AC, Cereser K, da Costa S, Santin A, Yatham LN, Kapczinski F: Traumatic life events in bipolar disorder: impact on BDNF levels and psychopathology. Bipolar Disord 2007;9(suppl 1):128-135.

39 Kapczinski F, Dias VV, Frey BN, KauerSant'Anna M: Brain-derived neurotrophic factor in bipolar disorder: beyond trait and state: comment on 'Decreased levels of serum brain-derived neurotrophic factor in both depressed and euthymic patients with unipolar depression and in euthymic patients with bipolar I and II disorders'. Bipolar Disord 2009;11:221-222.

40 Molendijk ML, Bus BA, Spinhoven P, Penninx BW, Kenis G, Prickaerts J, Voshaar RO, Elzinga BM: Serum levels of brain-derived neurotrophic factor in major depressive disorder: state-trait issues, clinical features and pharmacological treatment. Mol Psychiatry 2011;16:1088-1095.

41 Karege F, Schwald M, Cisse M: Postnatal developmental profile of brain-derived neurotrophic factor in rat brain and platelets. Neurosci Lett 2002;328:261-264.
42 Lang UE, Hellweg R, Seifert F, Schubert F, Gallinat J: Correlation between serum brainderived neurotrophic factor level and an in vivo marker of cortical integrity. Biol Psychiatry 2007;62:530-535.

43 Pardridge WM, Wu D, Sakane T: Combined use of carboxyl-directed protein pegylation and vector-mediated blood-brain barrier drug delivery system optimizes brain uptake of brain-derived neurotrophic factor following intravenous administration. Pharm Res 1998;15:576-582.

44 Schulte-Herbruggen O, Nassenstein C, Lommatzsch M, Quarcoo D, Renz H, Braun A: Tumor necrosis factor-alpha and interleukin-6 regulate secretion of brain-derived neurotrophic factor in human monocytes. J Neuroimmunol 2005;160:204-209.

45 Kerschensteiner M, Gallmeier E, Behrens L, Leal VV, Misgeld T, Klinkert WE, Kolbeck R, Hoppe E, Oropeza-Wekerle RL, Bartke I, Stadelmann C, Lassmann H, Wekerle H, Hohlfeld R: Activated human T cells, B cells, and monocytes produce brain-derived neurotrophic factor in vitro and in inflammatory brain lesions: a neuroprotective role of inflammation? J Exp Med 1999;189:865870.

46 Muller N, Myint AM, Schwarz MJ: Inflammatory biomarkers and depression. Neurotox Res 2011;19:308-318.

-47 Nagahara AH, Tuszynski MH: Potential therapeutic uses of BDNF in neurological and psychiatric disorders. Nat Rev Drug Discov 2011;10:209-219.
48 Greenberg ME, Xu B, Lu B, Hempstead BL: New insights in the biology of BDNF synthesis and release: implications in CNS function. J Neurosci 2009;29:12764-12767.

49 Karege F, Bondolfi G, Gervasoni N, Schwald M, Aubry JM, Bertschy G: Low brain-derived neurotrophic factor (BDNF) levels in serum of depressed patients probably result from lowered platelet BDNF release unrelated to platelet reactivity. Biol Psychiatry 2005;57: 1068-1072.

-50 Parakh K, Sakhuja A, Bhat U, Ziegelstein RC Platelet function in patients with depression. South Med J 2008;101:612-617.

-51 Lederbogen F, Baranyai R, Gilles M, MenartHoutermans B, Tschoepe D, Deuschle M: Effect of mental and physical stress on platelet activation markers in depressed patients and healthy subjects: a pilot study. Psychiatry Res 2004;127:55-64.

-52 Hansson AC, Cintra A, Belluardo N, Sommer W, Bhatnagar M, Bader M, Ganten D, Fuxe K: Gluco- and mineralocorticoid receptor-mediated regulation of neurotrophic factor gene expression in the dorsal hippocampus and the neocortex of the rat. Eur J Neurosci 2000;12:2918-2934.

53 Tadic A, Wagner S, Schlicht KF, Peetz D, Borysenko L, Dreimuller N, Hiemke C, Lieb K: The early non-increase of serum BDNF predicts failure of antidepressant treatment in patients with major depression: a pilot study. Prog Neuropsychopharmacol Biol Psychiatry 2011;35:415-420. 